\title{
Difference and Difference Quotient. Part IV
}

\author{
Xiquan Liang \\ Qingdao University of Science \\ and Technology \\ China
}

\author{
Ling Tang \\ Qingdao University of Science \\ and Technology \\ China
}

\author{
Xichun Jiang \\ Qingdao University of Science \\ and Technology \\ China
}

Summary. In this article, we give some important theorems of forward difference, backward difference, central difference and difference quotient and forward difference, backward difference, central difference and difference quotient formulas of some special functions.

MML identifier: $\underline{\mathrm{DIFF}_{-}}$, version: $\underline{7.11 .07 \quad 4.156 .1112}$

The papers [2], [7], [13], [3], [1], [6], [9], [4], [14], [8], [5], [15], [11], [12], and [10] provide the notation and terminology for this paper.

We adopt the following rules: $n$ denotes an element of $\mathbb{N}, h, k, x, x_{0}, x_{1}, x_{2}$, $x_{3}$ denote real numbers, and $f, g$ denote functions from $\mathbb{R}$ into $\mathbb{R}$.

Next we state a number of propositions:

(1) If $x_{0}>0$ and $x_{1}>0$, then $\log _{e} x_{0}-\log _{e} x_{1}=\log _{e}\left(\frac{x_{0}}{x_{1}}\right)$.

(2) If $x_{0}>0$ and $x_{1}>0$, then $\log _{e} x_{0}+\log _{e} x_{1}=\log _{e}\left(x_{0} \cdot x_{1}\right)$.

(3) If $x>0$, then $\log _{e} x=($ the function $\ln )(x)$.

(4) If $x_{0}>0$ and $x_{1}>0$, then (the function $\left.\ln \right)\left(x_{0}\right)-($ the function $\ln )\left(x_{1}\right)=$ (the function $\ln )\left(\frac{x_{0}}{x_{1}}\right)$.

(5) Suppose for every $x$ holds $f(x)=\frac{k}{x^{2}}$ and $x_{0} \neq 0$ and $x_{1} \neq 0$ and $x_{2} \neq 0$ and $x_{3} \neq 0$ and $x_{0}, x_{1}, x_{2}, x_{3}$ are mutually different. Then $\Delta[f]\left(x_{0}, x_{1}, x_{2}, x_{3}\right)=\frac{k \cdot\left(\frac{1}{x_{1} \cdot x_{2} \cdot x_{0}} \cdot\left(\frac{1}{x_{0}}+\frac{1}{x_{2}}+\frac{1}{x_{1}}\right)-\frac{1}{x_{2} \cdot x_{1} \cdot x_{3}} \cdot\left(\frac{1}{x_{3}}+\frac{1}{x_{1}}+\frac{1}{x_{2}}\right)\right)}{x_{0}-x_{3}}$. 
(6) Suppose $x_{0} \in$ dom(the function cot) and $x_{1} \in$ dom(the function cot). Then $\Delta[$ (the function cot) (the function cot) $]\left(x_{0}, x_{1}\right)=$ $-\frac{\left(\cos x_{1}\right)^{2}-\left(\cos x_{0}\right)^{2}}{\left(\sin x_{0} \cdot \sin x_{1}\right)^{2} \cdot\left(x_{0}-x_{1}\right)}$.

(7) Suppose $x \in$ dom (the function cot) and $x+h \in$ dom(the function cot). Then $\left(\Delta_{h}[(\right.$ the function cot) (the function cot $\left.)]\right)(x)=$ $\frac{\frac{1}{2} \cdot(\cos (2 \cdot(x+h))-\cos (2 \cdot x))}{(\sin (x+h) \cdot \sin x)^{2}}$.

(8) Suppose $x \in \operatorname{dom}($ the function cot) and $x-h \in$ dom(the function cot). Then $\left(\nabla_{h}[(\right.$ the function cot) (the function cot $\left.)]\right)(x)=$ $\frac{\frac{1}{2} \cdot(\cos (2 \cdot x)-\cos (2 \cdot(h-x)))}{(\sin x \cdot \sin (x-h))^{2}}$.

(9) Suppose $x+\frac{h}{2} \in$ dom(the function cot) and $x-\frac{h}{2} \in$ dom (the function cot). Then $\left(\delta_{h}[(\right.$ the function cot) (the function cot $\left.)]\right)(x)=$ $\frac{\frac{1}{2} \cdot(\cos (h+2 \cdot x)-\cos (h-2 \cdot x))}{\left(\sin \left(x+\frac{h}{2}\right) \cdot \sin \left(x-\frac{h}{2}\right)\right)^{2}}$.

(10) If $x_{0}, x_{1} \in$ dom cosec, then

$\Delta[\operatorname{cosec} \operatorname{cosec}]\left(x_{0}, x_{1}\right)=\frac{4 \cdot\left(\sin \left(x_{1}+x_{0}\right) \cdot \sin \left(x_{1}-x_{0}\right)\right)}{\left(\cos \left(x_{0}+x_{1}\right)-\cos \left(x_{0}-x_{1}\right)\right)^{2} \cdot\left(x_{0}-x_{1}\right)}$.

(11) If $x, x+h \in$ dom cosec, then $\left(\Delta_{h}[\operatorname{cosec} \operatorname{cosec}]\right)(x)=-\frac{4 \cdot \sin (2 \cdot x+h) \cdot \sin h}{(\cos (2 \cdot x+h)-\cos h)^{2}}$.

(12) If $x, x-h \in$ dom cosec, then $\left(\nabla_{h}[\operatorname{cosec} \operatorname{cosec}]\right)(x)=-\frac{4 \cdot \sin (2 \cdot x-h) \cdot \sin h}{(\cos (2 \cdot x-h)-\cos h)^{2}}$.

(13) If $x+\frac{h}{2}, x-\frac{h}{2} \in$ dom cosec, then $\left(\delta_{h}[\operatorname{cosec} \operatorname{cosec}]\right)(x)=-\frac{4 \cdot \sin (2 \cdot x) \cdot \sin h}{(\cos (2 \cdot x)-\cos h)^{2}}$.

(14) If $x_{0}, x_{1} \in$ dom sec, then

$\Delta[\sec \sec ]\left(x_{0}, x_{1}\right)=\frac{4 \cdot\left(\sin \left(x_{0}+x_{1}\right) \cdot \sin \left(x_{0}-x_{1}\right)\right)}{\left(\cos \left(x_{0}+x_{1}\right)+\cos \left(x_{0}-x_{1}\right)\right)^{2} \cdot\left(x_{0}-x_{1}\right)}$.

(15) If $x, x+h \in$ dom sec, then $\left(\Delta_{h}[\sec \sec ]\right)(x)=\frac{4 \cdot \sin (2 \cdot x+h) \cdot \sin h}{(\cos (2 \cdot x+h)+\cos h)^{2}}$.

(16) If $x, x-h \in$ dom sec, then $\left(\nabla_{h}[\sec \sec ]\right)(x)=\frac{4 \cdot \sin (2 \cdot x-h) \cdot \sin h}{(\cos (2 \cdot x-h)+\cos h)^{2}}$.

(17) If $x+\frac{h}{2}, x-\frac{h}{2} \in \operatorname{dom} \sec$, then $\left(\delta_{h}[\sec \sec ]\right)(x)=\frac{4 \cdot \sin (2 \cdot x) \cdot \sin h}{(\cos (2 \cdot x)+\cos h)^{2}}$.

(18) If $x_{0}, x_{1} \in$ dom cosec $\cap$ dom sec, then $\Delta[\operatorname{cosec} \sec ]\left(x_{0}, x_{1}\right)=$ $\frac{4 \cdot\left(\cos \left(x_{1}+x_{0}\right) \cdot \sin \left(x_{1}-x_{0}\right)\right)}{\sin \left(2 \cdot x_{0}\right) \cdot \sin \left(2 \cdot x_{1}\right)}$

(19) If $x+h, x \in$ dom cosec $\cap$ dom sec, then $\left(\Delta_{h}[\operatorname{cosec}\right.$ sec $\left.]\right)(x)=$ $-4 \cdot \frac{\cos (2 \cdot x+h) \cdot \sin h}{\sin (2 \cdot(x+h)) \cdot \sin (2 \cdot x)}$

(20) If $x-h, x \in \operatorname{dom} \operatorname{cosec} \cap$ dom sec, then $\left(\nabla_{h}[\operatorname{cosec}\right.$ sec $\left.]\right)(x)=$ $-4 \cdot \frac{\cos (2 \cdot x-h) \cdot \sin h}{\sin (2 \cdot x) \cdot \sin (2 \cdot(x-h))}$.

(21) If $x+\frac{h}{2}, x-\frac{h}{2} \in \operatorname{dom} \operatorname{cosec} \cap \operatorname{domsec}$, then $\left(\delta_{h}[\operatorname{cosec} \sec ]\right)(x)=$ $-4 \cdot \frac{\cos (2 \cdot x) \cdot \sin h}{\sin (2 \cdot x+h) \cdot \sin (2 \cdot x-h)}$.

(22) Suppose $x_{0} \in \operatorname{dom}$ (the function $\tan$ ) and $x_{1} \in \operatorname{dom}($ the function $\tan$ ). Then $\Delta[$ (the function tan) (the function tan) (the function $\cos )]\left(x_{0}, x_{1}\right)=$ $\Delta[$ (the function $\tan )$ (the function $\sin )]\left(x_{0}, x_{1}\right)$.

(23) Suppose $x \in \operatorname{dom}$ (the function tan) and $x+h \in \operatorname{dom}$ (the function tan). Then $\left(\Delta_{h}[(\right.$ the function tan) $($ the function $\tan )$ (the function $\left.\cos )]\right)(x)=$ 
$(($ the function $\tan )$ (the function $\sin ))(x+h)-(($ the function tan) (the function $\sin ))(x)$.

(24) Suppose $x \in$ dom(the function tan) and $x-h \in$ dom(the function $\tan )$. Then $\left(\nabla_{h}[(\right.$ the function tan) (the function tan) (the function $\cos )])(x)=(($ the function tan $)$ (the function $\sin ))(x)-(($ the function tan $)$ (the function $\sin ))(x-h)$.

(25) Suppose $x+\frac{h}{2} \in \operatorname{dom}$ (the function $\tan$ ) and $x-\frac{h}{2} \in$ dom (the function $\tan )$. Then $\left(\delta_{h}[\right.$ (the function $\tan )$ (the function $\left.\tan \right)$ (the function $\cos )])(x)=(($ the function tan $)$ (the function $\sin ))\left(x+\frac{h}{2}\right)-(($ the function $\tan )($ the function $\sin ))\left(x-\frac{h}{2}\right)$.

(26) Suppose $x_{0} \in$ dom (the function cot) and $x_{1} \in \operatorname{dom}$ (the function cot). Then $\Delta[$ (the function cot) (the function cot) (the function $\sin )]\left(x_{0}, x_{1}\right)=$ $\Delta[$ (the function cot) (the function $\cos )]\left(x_{0}, x_{1}\right)$.

(27) Suppose $x \in \operatorname{dom}$ (the function cot) and $x+h \in \operatorname{dom}$ (the function cot). Then $\left(\Delta_{h}[(\right.$ the function cot) (the function cot) (the function $\left.\sin )]\right)(x)=$ $(($ the function cot) (the function $\cos ))(x+h)-(($ the function cot) (the function $\cos ))(x)$.

(28) Suppose $x \in$ dom(the function cot) and $x-h \in$ dom(the function cot). Then $\left(\nabla_{h}[\right.$ (the function cot) (the function cot) (the function $\sin )])(x)=(($ the function cot $)$ (the function $\cos ))(x)-(($ the function cot $)$ (the function $\cos ))(x-h)$.

(29) Suppose $x+\frac{h}{2} \in \operatorname{dom}$ (the function cot) and $x-\frac{h}{2} \in$ dom (the function cot). Then $\left(\delta_{h}[\right.$ (the function cot) (the function cot) (the function $\sin )])(x)=(($ the function cot $)$ (the function $\cos ))\left(x+\frac{h}{2}\right)-(($ the function cot) (the function $\cos ))\left(x-\frac{h}{2}\right)$.

(30) If $x_{0}>0$ and $x_{1}>0$, then $\Delta$ the function $\left.\ln \right]\left(x_{0}, x_{1}\right)=$ $\frac{\text { (the function } \ln )\left(\frac{x_{0}}{x_{1}}\right)}{x_{0}-x_{1}}$.

(31) If $x>0$ and $x+h>0$, then $\left(\Delta_{h}[\right.$ the function $\left.\ln ]\right)(x)=($ the function $\ln )\left(1+\frac{h}{x}\right)$.

(32) If $x>0$ and $x-h>0$, then $\left(\nabla_{h}[\right.$ the function $\left.\ln ]\right)(x)=($ the function $\ln )\left(1+\frac{h}{x-h}\right)$.

(33) If $x+\frac{h}{2}>0$ and $x-\frac{h}{2}>0$, then $\left(\delta_{h}[\right.$ the function $\left.\ln ]\right)(x)=($ the function $\ln )\left(1+\frac{h}{x-\frac{h}{2}}\right)$.

(34) For all real numbers $h, k$ holds $\exp (h-k)=\frac{\exp h}{\exp k}$.

(35) $\left(\Delta_{h}[f]\right)(x)=(\operatorname{Shift}(f, h))(x)-f(x)$.

(36) If for every $x$ holds $f(x)=\left(\Delta_{h}[g]\right)(x)$, then $\Delta[f]\left(x_{0}, x_{1}\right)=\Delta[g]\left(x_{0}+\right.$ $\left.h, x_{1}+h\right)-\Delta[g]\left(x_{0}, x_{1}\right)$.

(37) $\left(\Delta_{h}\left[\Delta_{h}[f]\right]\right)(x)=\left(\Delta_{2 \cdot h}[f]\right)(x)-2 \cdot\left(\Delta_{h}[f]\right)(x)$.

(38) $\left(\nabla_{h}\left[\Delta_{h}[f]\right]\right)(x)=\left(\Delta_{h}[f]\right)(x)-\left(\nabla_{h}[f]\right)(x)$. 
(39) $\left(\delta_{h}\left[\Delta_{h}[f]\right]\right)(x)=\left(\Delta_{h}[f]\right)\left(x+\frac{h}{2}\right)-\left(\delta_{h}[f]\right)(x)$.

(40) $\quad\left(\vec{\Delta}_{h}[f]\right)(1)(x)=\left(\vec{\Delta}_{h}[f]\right)(0)(x+h)-\left(\vec{\Delta}_{h}[f]\right)(0)(x)$.

(41) $\left(\vec{\Delta}_{h}[f]\right)(n+1)(x)=\left(\vec{\Delta}_{h}[f]\right)(n)(x+h)-\left(\vec{\Delta}_{h}[f]\right)(n)(x)$.

(42) $\quad\left(\nabla_{h}[f]\right)(x)=f(x)-(\operatorname{Shift}(f,-h))(x)$.

(43) If for every $x$ holds $f(x)=\left(\nabla_{h}[g]\right)(x)$, then $\Delta[f]\left(x_{0}, x_{1}\right)=\Delta[g]\left(x_{0}, x_{1}\right)-$ $\Delta[g]\left(x_{0}-h, x_{1}-h\right)$.

(44) $\quad\left(\Delta_{h}\left[\nabla_{h}[f]\right]\right)(x)=\left(\Delta_{h}[f]\right)(x)-\left(\nabla_{h}[f]\right)(x)$.

(45) $\quad\left(\nabla_{h}\left[\nabla_{h}[f]\right]\right)(x)=2 \cdot\left(\nabla_{h}[f]\right)(x)-\left(\nabla_{2 \cdot h}[f]\right)(x)$.

(46) $\quad\left(\delta_{h}\left[\nabla_{h}[f]\right]\right)(x)=\left(\delta_{h}[f]\right)(x)-\left(\nabla_{h}[f]\right)\left(x-\frac{h}{2}\right)$.

(47) $\quad\left(\vec{\nabla}_{h}[f]\right)(1)(x)=\left(\vec{\nabla}_{h}[f]\right)(0)(x)-\left(\vec{\nabla}_{h}[f]\right)(0)(x-h)$.

(48) $\left(\vec{\nabla}_{h}[f]\right)(n+1)(x)=\left(\vec{\nabla}_{h}[f]\right)(n)(x)-\left(\vec{\nabla}_{h}[f]\right)(n)(x-h)$.

(49) $\left(\delta_{h}[f]\right)(x)=\left(\operatorname{Shift}\left(f, \frac{h}{2}\right)\right)(x)-\left(\operatorname{Shift}\left(f,-\frac{h}{2}\right)\right)(x)$.

(50) If for every $x$ holds $f(x)=\left(\delta_{h}[g]\right)(x)$, then $\Delta[f]\left(x_{0}, x_{1}\right)=\Delta[g]\left(x_{0}+\right.$ $\left.\frac{h}{2}, x_{1}+\frac{h}{2}\right)-\Delta[g]\left(x_{0}-\frac{h}{2}, x_{1}-\frac{h}{2}\right)$.

(51) $\quad\left(\Delta_{h}\left[\delta_{h}[f]\right]\right)(x)=\left(\Delta_{h}[f]\right)\left(x+\frac{h}{2}\right)-\left(\delta_{h}[f]\right)(x)$.

(52) $\left(\nabla_{h}\left[\delta_{h}[f]\right]\right)(x)=\left(\delta_{h}[f]\right)(x)-\left(\nabla_{h}[f]\right)\left(x-\frac{h}{2}\right)$.

(53) $\quad\left(\delta_{h}\left[\delta_{h}[f]\right]\right)(x)=\left(\Delta_{h}[f]\right)(x)-\left(\nabla_{h}[f]\right)(x)$.

(54) $\quad\left(\vec{\delta}_{h}[f]\right)(1)(x)=\left(\vec{\delta}_{h}[f]\right)(0)\left(x+\frac{h}{2}\right)-\left(\vec{\delta}_{h}[f]\right)(0)\left(x-\frac{h}{2}\right)$.

(55) $\quad\left(\vec{\delta}_{h}[f]\right)(n+1)(x)=\left(\vec{\delta}_{h}[f]\right)(n)\left(x+\frac{h}{2}\right)-\left(\vec{\delta}_{h}[f]\right)(n)\left(x-\frac{h}{2}\right)$.

(56) Suppose $x_{0} \in \operatorname{dom}$ (the function tan) and $x_{1} \in \operatorname{dom}$ (the function tan). Then $\Delta[($ the function $\tan )$ (the function $\tan )$ (the function sin $)]\left(x_{0}, x_{1}\right)=$ $\frac{\left(\sin x_{0}\right)^{3} \cdot\left(\cos x_{1}\right)^{2}-\left(\sin x_{1}\right)^{3} \cdot\left(\cos x_{0}\right)^{2}}{\left(\cos x_{0}\right)^{2} \cdot\left(\cos x_{1}\right)^{2} \cdot\left(x_{0}-x_{1}\right)}$.

(57) Suppose $x \in$ dom(the function tan) and $x+h \in$ dom(the function $\tan )$. Then $\left(\Delta_{h}[(\right.$ the function tan) (the function tan) (the function $\sin )])(x)=($ the function $\sin )(x+h)^{3} \cdot\left((\text { the function } \cos )(x+h)^{-1}\right)^{2}-$ (the function $\sin )(x)^{3} \cdot\left((\text { the function } \cos )(x)^{-1}\right)^{2}$.

(58) Suppose $x \in \operatorname{dom}$ (the function tan) and $x-h \in$ dom(the function $\tan )$. Then $\left(\nabla_{h}[(\right.$ the function $\tan )$ (the function $\tan )$ (the function $\sin )])(x)=($ the function $\sin )(x)^{3} \cdot\left((\text { the function } \cos )(x)^{-1}\right)^{2}-$ (the function $\sin )(x-h)^{3} \cdot\left((\text { the function } \cos )(x-h)^{-1}\right)^{2}$.

(59) Suppose $x+\frac{h}{2} \in \operatorname{dom}$ (the function $\tan$ ) and $x-\frac{h}{2} \in$ dom (the function $\tan )$. Then $\left(\delta_{h}[\right.$ (the function $\tan )$ (the function $\left.\tan \right)$ (the function $\sin )])(x)=($ the function $\sin )\left(x+\frac{h}{2}\right)^{3} \cdot\left((\text { the function } \cos )\left(x+\frac{h}{2}\right)^{-1}\right)^{2}-$ (the function $\sin )\left(x-\frac{h}{2}\right)^{3} \cdot\left((\text { the function } \cos )\left(x-\frac{h}{2}\right)^{-1}\right)^{2}$.

(60) Suppose $x_{0} \in \operatorname{dom}$ (the function cot) and $x_{1} \in \operatorname{dom}$ (the function cot). Then $\Delta[$ (the function cot) (the function cot) (the function cos) $]\left(x_{0}, x_{1}\right)=$ $\frac{\left(\cos x_{0}\right)^{3} \cdot\left(\sin x_{1}\right)^{2}-\left(\cos x_{1}\right)^{3} \cdot\left(\sin x_{0}\right)^{2}}{\left(\sin x_{0}\right)^{2} \cdot\left(\sin x_{1}\right)^{2} \cdot\left(x_{0}-x_{1}\right)}$. 
(61) Suppose $x \in \operatorname{dom}($ the function $\cot$ ) and $x+h \in$ dom(the function cot). Then $\left(\Delta_{h}[\right.$ (the function cot) (the function cot) (the function $\cos )])(x)=($ the function $\cos )(x+h)^{3} \cdot\left((\text { the function } \sin )(x+h)^{-1}\right)^{2}-$ (the function $\cos )(x)^{3} \cdot\left((\text { the function } \sin )(x)^{-1}\right)^{2}$.

(62) Suppose $x \in \operatorname{dom}($ the function $\cot$ ) and $x-h \in$ dom(the function cot). Then $\left(\nabla_{h}[\right.$ (the function cot) (the function cot) (the function $\cos )])(x)=($ the function $\cos )(x)^{3} \cdot\left((\text { the function } \sin )(x)^{-1}\right)^{2}-$ (the function $\cos )(x-h)^{3} \cdot\left((\text { the function } \sin )(x-h)^{-1}\right)^{2}$.

(63) Suppose $x+\frac{h}{2} \in \operatorname{dom}$ (the function cot) and $x-\frac{h}{2} \in$ dom (the function cot). Then $\left(\delta_{h}[\right.$ (the function cot) (the function cot) (the function $\cos )])(x)=($ the function $\cos )\left(x+\frac{h}{2}\right)^{3} \cdot\left((\text { the function } \sin )\left(x+\frac{h}{2}\right)^{-1}\right)^{2}-$ (the function $\cos )\left(x-\frac{h}{2}\right)^{3} \cdot\left((\text { the function } \sin )\left(x-\frac{h}{2}\right)^{-1}\right)^{2}$.

\section{REFERENCES}

[1] Grzegorz Bancerek. The fundamental properties of natural numbers. Formalized Mathematics, 1(1):41-46, 1990.

[2] Czesław Byliński. The complex numbers. Formalized Mathematics, 1(3):507-513, 1990.

[3] Czesław Byliński. Functions from a set to a set. Formalized Mathematics, 1(1):153-164, 1990.

[4] Krzysztof Hryniewiecki. Basic properties of real numbers. Formalized Mathematics, 1(1):35-40, 1990.

[5] Jarosław Kotowicz. Real sequences and basic operations on them. Formalized Mathematics, 1(2):269-272, 1990.

[6] Rafał Kwiatek. Factorial and Newton coefficients. Formalized Mathematics, 1(5):887-890, 1990.

[7] Bo Li, Yan Zhang, and Xiquan Liang. Difference and difference quotient. Formalized Mathematics, 14(3):115-119, 2006, doi:10.2478/v10037-006-0014-z.

[8] Beata Perkowska. Functional sequence from a domain to a domain. Formalized Mathematics, 3(1):17-21, 1992.

[9] Konrad Raczkowski and Andrzej Nędzusiak. Real exponents and logarithms. Formalized Mathematics, 2(2):213-216, 1991.

[10] Yasunari Shidama. The Taylor expansions. Formalized Mathematics, 12(2):195-200, 2004.

[11] Andrzej Trybulec and Czesław Byliński. Some properties of real numbers. Formalized Mathematics, 1(3):445-449, 1990.

[12] Zinaida Trybulec. Properties of subsets. Formalized Mathematics, 1(1):67-71, 1990.

[13] Peng Wang and Bo Li. Several differentiation formulas of special functions. Part V. Formalized Mathematics, 15(3):73-79, 2007, doi:10.2478/v10037-007-0009-4.

[14] Edmund Woronowicz. Relations defined on sets. Formalized Mathematics, 1(1):181-186, 1990.

[15] Yuguang Yang and Yasunari Shidama. Trigonometric functions and existence of circle ratio. Formalized Mathematics, 7(2):255-263, 1998.

Received July 12, 2010 